\title{
CVaR Robust Mean-CVaR Portfolio Optimization
}

\author{
Maziar Salahi, Farshid Mehrdoust, and Farzaneh Piri \\ Department of Applied Mathematics, Faculty of Mathematical Sciences, University of Guilan, Namjoo Street, P.O. Box 1914, Rasht, Iran
}

Correspondence should be addressed to Maziar Salahi; salahim@guilan.ac.ir

Received 16 July 2013; Accepted 16 August 2013

Academic Editors: X. Liu and Q. Song

Copyright (C) 2013 Maziar Salahi et al. This is an open access article distributed under the Creative Commons Attribution License, which permits unrestricted use, distribution, and reproduction in any medium, provided the original work is properly cited.

\begin{abstract}
One of the most important problems faced by every investor is asset allocation. An investor during making investment decisions has to search for equilibrium between risk and returns. Risk and return are uncertain parameters in the suggested portfolio optimization models and should be estimated to solve the problem. However, the estimation might lead to large error in the final decision. One of the widely used and effective approaches for optimization with data uncertainty is robust optimization. In this paper, we present a new robust portfolio optimization technique for mean- $C V a R$ portfolio selection problem under the estimation risk in mean return. We additionally use $C V a R$ as risk measure, to measure the estimation risk in mean return. To solve the model efficiently, we use the smoothing technique of Alexander et al. (2006). We compare the performance of the CVaR robust mean-CVaR model with robust mean-CVaR models using interval and ellipsoidal uncertainty sets. It is observed that the $C V a R$ robust mean-CVaR portfolios are more diversified. Moreover, we study the impact of the value of confidence level on the conservatism level of a portfolio and also on the value of the maximum expected return of the portfolio.
\end{abstract}

\section{Introduction}

Portfolio optimization is one of the best known approaches in financial portfolio selection. The earliest technique to solve the portfolio selection problem is developed by Harry Markowitz in the 1952. In his so-called mean-variance (MV) portfolio optimization model, the portfolio return is measured by the expected return of the portfolio, and the associated risk is measured by the variance of portfolio returns [1].

Variance as the risk measure has its weaknesses. Controlling the variance does not only lead to low deviation from the expected return on the downside, but also on the upside [2]. Hence, alternative risk measures have been suggested to replace the variance such as Value at Risk ( $V a R)$ that manage and control risk in terms of percentiles of loss distribution. Instead of regarding both upside and downside of the expected return, $V a R$ considers only the downside of the expected return as risk and represents the predicted maximum loss with a specified confidence level (e.g., 95\%) over a certain period of time (e.g., one day) [3-5].

$\mathrm{VaR}$ is a popular risk measure. However, $\mathrm{VaR}$ may have drawbacks and undesirable properties that limit its use [68], such as lack of subadditivity; that is, $V a R$ of two different investment portfolios may be greater than the sum of the individual $\mathrm{VaRs}$. Also, $\mathrm{VaR}$ is nonconvex and nonsmooth and has multiple local minimum, while we seek the global minimum $[4,8,9]$. So alternative risk measures were introduced such as Conditional Value at Risk $(\mathrm{CVaR})$ - the conditional expected value of loss, under the condition that it exceeds the value at risk [3]. VaR implies that "what is the maximum loss that we realize?" but $C V a R$ asks: "How do we expect to incur losses when situation is undesirable?". Numerical experiments show that minimum $C V a R$ often leads to optimal solutions close to the minimum $V a R$, because $V a R$ never exceeds $C V a R$ [5]. $C V a R$ has better properties than $V a R$. It is a convex optimization problem, and thus it is easy to optimize [4]. It is demonstrated that linear programming techniques can be used for optimization of $C V a R$ risk measure $[5,9]$.

Risk and return are uncertain parameters in portfolio optimization models, and estimating them might lead to large error in the final decision. To deal with such situation, one of the widely used and effective approaches is robust optimization technique. In this paper, we have applied this technique to give the robust counterpart of the mean- $\mathrm{CVaR}$ portfolio selection problem under the estimation risk in mean return. Moreover, we have used $C V a R$ as the risk measure 
to measure the estimation risk in mean return. The rest of the paper is organized as follows. In Section 2, we state the mean-CVaR portfolio selection problem. Then because of the inevitable estimation error of the mean return of the assets, we present robust optimization by $C V a R$ in Section 3. To solve the model efficiently, we use the smoothing technique of Alexander et al. [10]. Finally, in Section 4, we compare the performance of the $C V a R$ robust mean-CVaR model with robust mean-CVaR models using interval and ellipsoidal uncertainty sets on an example. We have observed that the $C V a R$ robust mean- $C V a R$ portfolios are more diversified and they are sensitive to initial data used to generate each set of $\mu$ samples. Moreover, we demonstrate that the value of confidence level affects the conservatism level, diversification, and also the value of the maximum expected return of the resulting portfolios.

\section{Mean-Conditional Value at Risk}

Consider assets $S_{1}, \ldots, S_{n}, n \geq 2$, with random returns. Suppose $\mu_{i}$ denotes the expected return of asset $S_{i}$, and also consider $x_{i}$ as the proportion of holding in the $i$ th asset. We can represent the expected return of the resulting portfolio $x$ as follows:

$$
E[x]=\mu_{1} x_{1}+\cdots+\mu_{n} x_{n}=\mu^{T} x .
$$

Also, we will assume that the set of feasible portfolios is a nonempty polyhedral set and represent that as $\Omega=\{x \mid A x=$ $b, C x \geq d\}$ where $A$ is a $m \times n$ matrix, $b$ is an $m$-dimensional vector, $C$ is a $p \times n$ matrix, and $d$ is a $p$-dimensional vector [4]. In particular, one of the constraints in the set $\Omega$ is $\sum_{i=1}^{n} x_{i}=1$.

Let $f(x, y)$ denote the loss function when we choose the portfolio $x$ from a set of feasible portfolios, and $y$ is the realization of the random events (the vector of the asset returns of $n$ assets). We consider the portfolio return loss, $f(x, y)$, the negative of the portfolio return that is a convex (linear) function of the portfolio variables $x$ :

$$
f(x, y)=-y^{T} x=-\left[y_{1} x_{1}+\cdots+y_{n} x_{n}\right] .
$$

We assume that the random vector $y$ has a probability density function denoted by $p(y)$. For a fixed decision vector $x$, the cumulative distribution function of the loss associated with that vector is computed as follows:

$$
\psi(x, \gamma)=\int_{f(x, y) \leq \gamma} p(y) d y .
$$

Then, for a given confidence level $\alpha$, the $\alpha$-VaR associated with portfolio $x$ is represented as

$$
\operatorname{VaR}_{\alpha}(x)=\min \{\gamma \in \mathbb{R} \mid \psi(x, \gamma) \geq \alpha\} .
$$

Also, we define the $\alpha$-CVaR associated with portfolio $x$ as

$$
\operatorname{CVaR}_{\alpha}(x)=\frac{1}{(1-\alpha)} \int_{f(x, y) \geq \operatorname{VaR}_{\alpha}(x)} f(x, y) p(y) d y .
$$

Theorem 1. We always have $\mathrm{CVaR}_{\alpha}(x) \geq \operatorname{VaR}_{\alpha}(x)$, that means CVaR of a portfolio is always at least as big as its VaR.
Consequently, portfolios with small CVaR also have small $\mathrm{VaR}$. However, in general minimizing $C V a R$ and $V a R$ are not equivalent.

Proof. See [4].

Since the definition of $C V a R$ implies the $V a R$ function clearly, it is difficult to work with and optimize this function. Instead, the following simpler auxiliary function is considered [5]:

$$
F_{\alpha}(x, \gamma)=\gamma+\frac{1}{(1-\alpha)} \int_{f(x, y) \geq \gamma}(f(x, y)-\gamma) p(y) d y,
$$

and/or

$$
F_{\alpha}(x, \gamma)=\gamma+\frac{1}{(1-\alpha)} \int(f(x, y)-\gamma)^{+} p(y) d y,
$$

where $a^{+}=\max \{a, 0\}$. This function, considered as a function of $\gamma$, has the following important properties that makes it useful for the computation of $V a R$ and $C V a R$ [4]:

(1) $F_{\alpha}$ is a convex function of $\gamma$.

(2) $V a R_{\alpha}$ is a minimizer over $\gamma$ of $F_{\alpha}$.

(3) The minimum value over $\gamma$ of the function $F_{\alpha}$ is $\mathrm{CVaR}_{\alpha}$.

As a consequence of the listed properties, we immediately deduce that, in order to minimize $\operatorname{CVaR}_{\alpha}(x)$ over $x$, we need to minimize the function $F_{\alpha}(x, \gamma)$ with respect to $x$ and $\gamma$ simultaneously

$$
\min _{x} C V a R_{\alpha}(x)=\min _{x, \gamma} F_{\alpha}(x, \gamma) .
$$

Consequently, we can optimize $C V a R$ directly, without needing to compute $V a R$ first. Since we assumed that the loss function $f(x, y)$ is the convex (linear) function of the portfolio variables $x, F_{\alpha}(x, \gamma)$ is also a convex (linear) function of $x$. In this case, provided the feasible portfolio set $\Omega$ is also convex, the optimization problems in (8) are convex optimization problems that can be solved using well-known optimization techniques for such problems.

Instead of using the density function $p(y)$ of the random events in formulation (7) that it is often impossible or undesirable to compute it, we can use a number of scenarios in the names of $y_{i}$ for $i=1, \ldots, T$. In this case, we consider the following approximation to the function $F_{\alpha}(x, \gamma)$

$$
\bar{F}_{\alpha}(x, \gamma)=\gamma+\frac{1}{(1-\alpha) T} \sum_{i=1}^{T}\left(f\left(x, y_{i}\right)-\gamma\right)^{+} .
$$

Now, in the problem $\min _{x} C V a R_{\alpha}(x)$, we replace $F_{\alpha}(x, \gamma)$ with $\bar{F}_{\alpha}(x, \gamma)$

$$
\min _{x, \gamma} \gamma+\frac{1}{(1-\alpha) T} \sum_{i=1}^{T}\left(f\left(x, y_{i}\right)-\gamma\right)^{+} .
$$


To solve this optimization problem, we introduce artificial variables $z_{i}$ to replace $\left(f\left(x, y_{i}\right)-\gamma\right)^{+}$. To do so, we add the constraints $z_{i} \geq 0$ and $z_{i} \geq f\left(x, y_{i}\right)-\gamma$ to the problem [5]:

$$
\begin{array}{ll}
\min _{x, z, \gamma} & \gamma+\frac{1}{(1-\alpha) T} \sum_{i=1}^{T} z_{i} \\
\text { s.t. } & z_{i} \geq 0, \quad i=1, \ldots, T \\
& z_{i} \geq f\left(x, y_{i}\right)-\gamma, \quad i=1, \ldots, T \\
& x \in \Omega .
\end{array}
$$

It should be noted that risk managers often try to optimize risk measure while expected return is more than a threshold value. In this case, we can represent mean-CVaR model as follows:

$$
\begin{array}{ll}
\min _{x, z, \gamma} & \gamma+\frac{1}{(1-\alpha) T} \sum_{i=1}^{T} z_{i} \\
\text { s.t. } & \mu^{T} x \geq R, \\
& z_{i} \geq 0, \quad i=1, \ldots, T \\
& z_{i} \geq f\left(x, y_{i}\right)-\gamma, \quad i=1, \ldots, T \\
& x \in \Omega
\end{array}
$$

or

$$
\begin{array}{ll}
\min _{x, z, \gamma} & -\mu^{T} x+\lambda\left(\gamma+\frac{1}{(1-\alpha) T} \sum_{i=1}^{T} z_{i}\right) \\
\text { s.t. } & z_{i} \geq 0, \quad i=1, \ldots, T \\
& z_{i} \geq f\left(x, y_{i}\right)-\gamma, \quad i=1, \ldots, T \\
& x \in \Omega,
\end{array}
$$

where the first constraint of problem (12) indicates that the expected return is no less than the target value $R$ and $\lambda \geq 0$ used in problem (13) is risk aversion parameter that adapts the balance between expected return and $C V a R_{\alpha}(x)$. It is important to note that there is an equivalence between $R$ and $\lambda$ so that the problems (12) and (13) generate the same efficient frontiers. Since $f(x, y)$ is linear in $x$, all the expressions $z_{i} \geq f\left(x, y_{i}\right)-\gamma$ represent linear constraints, and therefore the problem is a linear programming problem that can be efficiently solved using the simplex or interior point methods.

\section{3. $C V a R$ Robust Mean-CVaR Model}

One of the uncertain parameters for mean-CVaR model is $\mu$, and using estimations for this parameter leads to an estimation risk in portfolio selection. In particular, small differences in the estimations of $\mu$ can create large changes in the composition of an optimal portfolio. One way to reduce the sensitivity of mean-CVaR model to the parameter estimations is using robust optimization to determine the optimal portfolio under the worst case scenario in the uncertainty set of the expected return. To this end, we represented robust mean-CVaR models with interval and ellipsoidal uncertainty sets in the previous studies that have been demonstrated in formulations (14) and (15), respectively,

$$
\begin{array}{ll}
\min _{x, z, \gamma} & \gamma+\frac{1}{(1-\alpha) T} \sum_{i=1}^{T} z_{i} \\
\text { s.t. } & \left(\mu^{L}\right)^{T} x \geq R, \\
& z_{i} \geq 0, \quad i=1, \ldots, T \\
& z_{i} \geq f\left(x, y_{i}\right)-\gamma, \quad i=1, \ldots, T \\
& x \in \Omega, \\
\min _{x, z, \gamma} \quad & \gamma+\frac{1}{(1-\alpha) T} \sum_{i=1}^{T} z_{i} \\
\text { s.t. } \quad & \left\|M^{T} x\right\| \leq \mu^{T} x-R, \\
& z_{i} \geq 0, \quad i=1, \ldots, T \\
& z_{i} \geq f\left(x, y_{i}\right)-\gamma, \quad i=1, \ldots, T \\
& x \in \Omega,
\end{array}
$$

where $\mu^{L}$ is a given vector and $M$ is a $n$-dimensional matrix.

Now, we present $C V a R$ robust mean-CVaR portfolio optimization problem that estimation risk in mean return is measured by $C V a R$. The $C V a R$ robust mean-CVaR model specifies that an optimal portfolio based on the tail of the mean loss distribution and the adjustment of the confidence level with regard to the preference of the investor corresponds to the adjustment of the conservative level, considering the uncertainty of the mean return [11].

In this model, $C V a R$ is used to measure the risk of the portfolio return as before. In addition, when using the mean$C V a R$ model, we consider the uncertainty of the expected return that can be considered as estimation risk and use $C V a R$ to measure estimation risk. $C V a R$ with this perspective is denoted as $C V a R^{\mu}$ (We use $C V a R^{y}$ to denote the $C V a R$ risk measure discussed in Section 2 in order to differentiate it from $C V a R^{\mu}$ and also we use $F_{\alpha}^{y}(x, \gamma)$ to denote its associated $\left.F_{\alpha}(x, \gamma)\right)$. Thus, considering the problem (13), a $C V a R$ robust mean-CVaR portfolio will be determined as the solution of the following optimization problem:

$$
\begin{array}{ll}
\min _{x, z, \gamma} & \operatorname{CVaR}_{\beta}^{\mu}\left(-\mu^{T} x\right)+\lambda\left(\gamma+\frac{1}{(1-\alpha) T} \sum_{i=1}^{T} z_{i}\right) \\
\text { s.t. } & z_{i} \geq 0, \quad i=1, \ldots, T \\
& z_{i} \geq f\left(x, y_{i}\right)-\gamma, \quad i=1, \ldots, T \\
& x \in \Omega .
\end{array}
$$

For a portfolio of $n$ assets, we assume $\mu \in \mathbb{R}^{n}$ is the random vector of the expected returns of the assets with a probability density function $p(\mu)$. To determine the mean 
TABLE 1: Mean return vector $\mu$.

\begin{tabular}{rcccccccr}
\hline $0.01 \times$ & S1 & S2 & S3 & S4 & S5 & S6 & S7 & S8 \\
\hline & 1.0160 & 0.4746 & 0.4756 & 0.4734 & 0.4742 & -0.0500 & -0.1120 & 0.0360 \\
\hline
\end{tabular}

TABLE 2: Covariance matrix $Q$.

\begin{tabular}{lccccccc}
\hline $0.01 \times$ & S1 & S2 & S3 & S4 & S5 & S6 & S7 \\
\hline S1 & 0.0980 & & & & & & \\
S2 & 0.0659 & 0.1549 & & & & & \\
S3 & 0.0714 & 0.0911 & 0.2738 & & & & \\
S4 & 0.0105 & 0.0058 & -0.0062 & 0.0097 & & & \\
S5 & 0.0058 & 0.0379 & -0.0116 & 0.0082 & 0.0461 & & \\
S6 & -0.0236 & -0.0260 & 0.0083 & -0.0215 & -0.0315 & 0.2691 & 0.0925 \\
S7 & -0.0164 & 0.0079 & 0.0059 & -0.0003 & 0.0076 & -0.0080 & -0.0095 \\
S8 & 0.0004 & -0.0248 & 0.0077 & -0.0026 & -0.0304 & 0.0159 & 0.0245 \\
\hline
\end{tabular}

loss of the portfolio, we define mean loss function, $f(x, \mu)$, as follows [11]:

$$
f(x, \mu)=-\mu^{T} x=-\left[\mu_{1} x_{1}+\cdots+\mu_{n} x_{n}\right] .
$$

So, for confidence level $\beta, \operatorname{CVaR}_{\beta}^{\mu}\left(-\mu^{T} x\right)$ can be defined as follows:

$$
\operatorname{CVaR}_{\beta}^{\mu}\left(-\mu^{T} x\right)=\min _{\eta}\left(\eta+\frac{1}{1-\beta} E\left([f(x, \mu)-\eta]^{+}\right)\right) .
$$

According to the definition of $C V a R_{\beta}^{\mu}$ and $C V a R$ robust mean-CVaR model, we find that $C V a R_{\beta}^{\mu}$ will increase as the value of $\beta$ increases. This corresponds to taking more pessimism on the estimation risk in $\mu$ in the model and to optimizing the portfolio under worse cases of the mean loss. Thus, the resulting $C V a R$ robust portfolio is more conservative. Conversely, conservatism of the portfolio is reduced as the value of $\beta$ decreases [11]. In Section 4, we will illustrate the impact of the value of $\beta$ on the conservatism level of a portfolio and also on the value of the maximum expected return of the portfolio.

As before, we can consider an auxiliary function to simplify the computations:

$$
F_{\beta}^{\mu}(x, \eta)=\eta+\frac{1}{(1-\beta)} \int_{\mu \in \mathbb{R}^{n}}(f(x, \mu)-\eta)^{+} p(\mu) d \mu,
$$

and use the following approximation to the function $F_{\beta}^{\mu}(x, \eta)$ :

$$
\bar{F}_{\beta}^{\mu}(x, \eta)=\eta+\frac{1}{(1-\beta) m} \sum_{i=1}^{m}\left(-\mu_{i}^{T} x-\eta\right)^{+},
$$

where $\mu_{1}, \ldots, \mu_{m}$ are a collection of $m$ independent samples for $\mu$ based on its density function $p(\mu)$. We can show that [5]

$$
\min _{x \in \Omega} \operatorname{CVaR} R_{\beta}^{\mu}\left(-\mu^{T} x\right)=\min _{x \in \Omega, \eta} \bar{F}_{\beta}^{\mu}(x, \eta) .
$$

So, with introducing artificial variables $v_{i}$ to replace $\left(f\left(x, \mu_{i}\right)-\eta\right)^{+}$in $\bar{F}_{\beta}^{\mu}(x, \eta)$ and adding the constraints $v_{i} \geq 0$ and $v_{i} \geq f\left(x, \mu_{i}\right)-\eta$ to the problem to do so, the $C V a R$ robust mean-CVaR portfolio optimization problem becomes

$$
\begin{array}{ll}
\min _{x, z, v, \gamma, \eta} \quad \eta+\frac{1}{(1-\beta) m} \sum_{i=1}^{m} v_{i}+\lambda\left(\gamma+\frac{1}{(1-\alpha) T} \sum_{i=1}^{T} z_{i}\right) \\
\text { s.t. } \quad z_{i} \geq 0, \quad i=1, \ldots, T \\
z_{i} \geq f\left(x, y_{i}\right)-\gamma, \quad i=1, \ldots, T \\
v_{i} \geq 0, \quad i=1, \ldots, m \\
v_{i} \geq f\left(x, \mu_{i}\right)-\eta, \quad i=1, \ldots, m \\
x & \in \Omega .
\end{array}
$$

This problem has $O(m+n+T)$ variables and $O(m+$ $n+T)$ constraints that $m$ is the number of $\mu$-samples, $n$ is the number of assets, and $T$ is the number of $y$-scenarios. When the number of $y$-scenarios and $\mu$-samples increase, the approximations are getting closer to the exact values. But the computational cost significantly increases and thus makes the method inefficient.

Instead of this method, we can more efficiently determine the $C V a R$ robust mean- $C V a R$ portfolios using the smoothing method suggested by Alexander et al. [10]. Alexander presented the following function to approximate $\bar{F}_{\beta}^{\mu}(x, \eta)$ :

$$
\widehat{F}_{\beta}^{\mu}(x, \eta)=\eta+\frac{1}{(1-\beta) m} \sum_{i=1}^{m} \rho_{\varepsilon}\left(-\mu_{i}^{T} x-\eta\right),
$$

where $\rho_{\varepsilon}(a)$ is defined as follows:

$$
\rho_{\varepsilon}(a)= \begin{cases}a & a \geq \varepsilon \\ \frac{a^{2}}{4 \varepsilon}+\frac{1}{2} a+\frac{1}{4} \varepsilon & -\varepsilon \leq a \leq \varepsilon \\ 0 & \text { o.w. }\end{cases}
$$

For a given resolution parameter $\varepsilon>0, \rho_{\varepsilon}(a)$ is continuous differentiable and approximates the piecewise linear 
TABLE 3: Time required to compute maximum-return $(\lambda=0)$ portfolios for LP and smoothing approach $(\beta=99 \%, \varepsilon=0.005)$.

\begin{tabular}{|c|c|c|c|c|c|c|c|}
\hline \multirow{2}{*}{ Scenarios $(\mathrm{T})$} & \multirow{2}{*}{ Samples (m) } & \multicolumn{3}{|c|}{ LP (problem (22)) } & \multicolumn{3}{|c|}{ Smoothing (problem (26)) } \\
\hline & & 8 assets & 50 assets & 148 assets & 8 assets & 50 assets & 148 assets \\
\hline 500 & 5,000 & 3.01 & 5.7 & 23.05 & 1.13 & 3.08 & 15.67 \\
\hline 1,000 & 10,000 & 4.85 & 9.95 & 37.2 & 1.28 & 4.67 & 26.1 \\
\hline 3,000 & 25,000 & 13.86 & 45.32 & 185.49 & 1.69 & 12.22 & 79.31 \\
\hline
\end{tabular}

function $\max (a, 0)$ [10]. We can also use this function to approximate $\bar{F}_{\alpha}^{y}(x, \gamma)$ as follows:

$$
\widehat{F}_{\alpha}^{y}(x, \gamma)=\gamma+\frac{1}{(1-\alpha) T} \sum_{i=1}^{T} \rho_{\varepsilon}\left(f\left(x, y_{i}\right)-\gamma\right) .
$$

Using smoothing method, the $C V a R$ robust mean-CVaR model is as follows:

$$
\begin{aligned}
\min _{x, \gamma, \eta} \eta & +\frac{1}{(1-\beta) m} \sum_{i=1}^{m} \rho_{\varepsilon}\left(-\mu_{i}^{T} x-\eta\right) \\
& +\lambda\left(\gamma+\frac{1}{(1-\alpha) T} \sum_{i=1}^{T} \rho_{\varepsilon}\left(-y_{i}^{T} x-\gamma\right)\right)
\end{aligned}
$$

s.t. $x \in \Omega$.

This formulation has $O(n)$ variables and $O(n)$ constraints. Thus, the number of variables and constraints does not change as the size of $\mu$-samples $(m)$ and $y$-scenarios $(T)$ increases. The efficiency of the smoothing approach is shown in Section 4.

\section{Numerical Results}

In this section, first we will compare the performance of the $C V a R$ robust mean-CVaR model with robust mean-CVaR models using interval and ellipsoidal uncertainty sets by actual data. Then, we will compare time required to compute the $C V a R$ robust portfolios using problems (22) and (26). The dataset used here contains available returns for eight assets that expected return [12] and covariance matrix of the return of assets which have been given in Tables 1 and 2 . In addition, the computations are based on 10,000 $\mu$-samples generated from the Monte Carlo resampling (RS) method introduced in [13] and $96 y$-scenarios obtained via computer simulation. It should be further noted that the computation is performed in MATLAB version 7.12, and run on a Core i5 CPU $2.40 \mathrm{GHz}$ Laptop with $4 \mathrm{~GB}$ of RAM. Problems are solved using CVX [14] and function "fmincon" in Optimization Toolbox of MATLAB.

4.1. Sensitivity to Initial Data. To show the sensitivity of the $C V a R$ robust portfolios to initial data, we repeat RS sampling technique 100 times. Each of Figures 1, 2 and 3 display 100 $\mathrm{CVaR}$ robust actual frontiers (actual frontiers are obtained by applying the true parameters on the portfolio weights derived from their estimated values [15]) for $\beta=99 \%, 90 \%, 75 \%$, respectively.

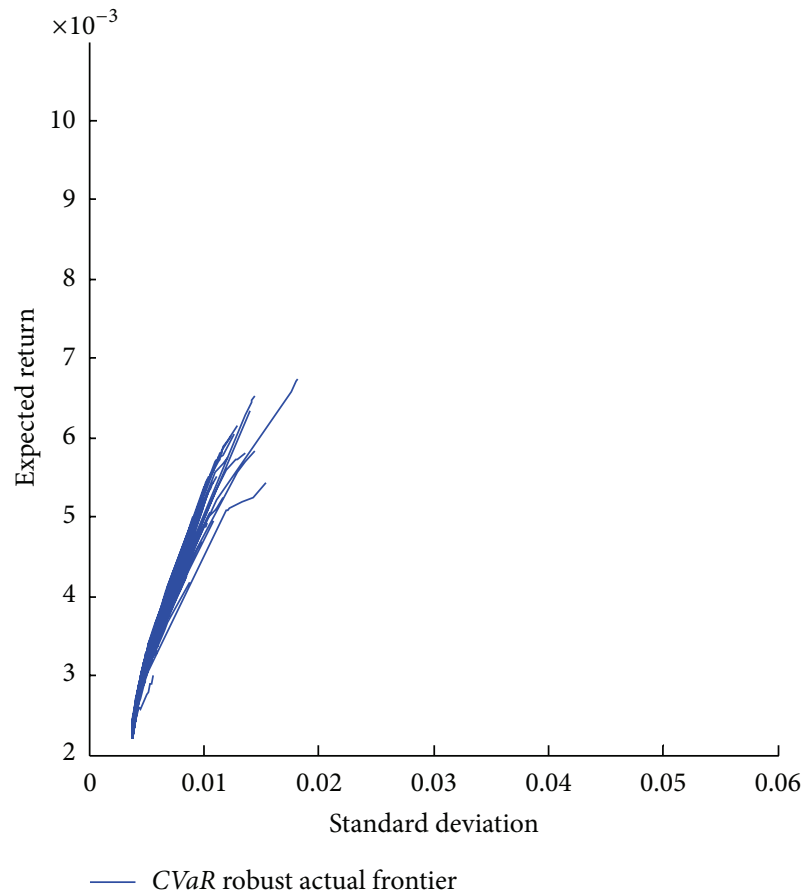

Figure 1: $100 C V a R$ robust mean-CVaR actual frontiers $(\beta=99 \%)$.

As can be seen from figures, the $C V a R$ robust mean$C V a R$ actual frontiers change with initial data used to generate $\mu$ samples. Also, this changes increase as the confidence level $\beta$ decreases. Thus, we can regard $\beta$ as an estimation risk aversion parameter. With these qualities, an investor who is more averse to estimation risk will choose a larger $\beta$. On the other hand, an investor who is more tolerant to estimation risk may choose a smaller $\beta$.

4.2. Portfolio Diversification. As we know, diversification decreases risk [4]. Portfolio diversification indicates distributing investment among assets in the portfolio. We illustrate in the following that compared with the robust mean$C V a R$ portfolios with interval and ellipsoidal uncertainty sets, the $C V a R$ robust mean-CVaR portfolios are more diversified. In addition, the diversification of the $C V a R$ robust mean-CVaR portfolios decreases as the confidence level $\beta$ decreases.

To do so, we compute the $C V a R$ robust mean-CVaR portfolios (for $\beta=99 \%, 90 \%, 75 \%$ ) and robust mean-CVaR portfolios with interval and ellipsoidal uncertainty sets for the 8-asset example. The composition graphs of the resulting optimal portfolios are presented in Figures 4, 5, 6, 7, and 8. 


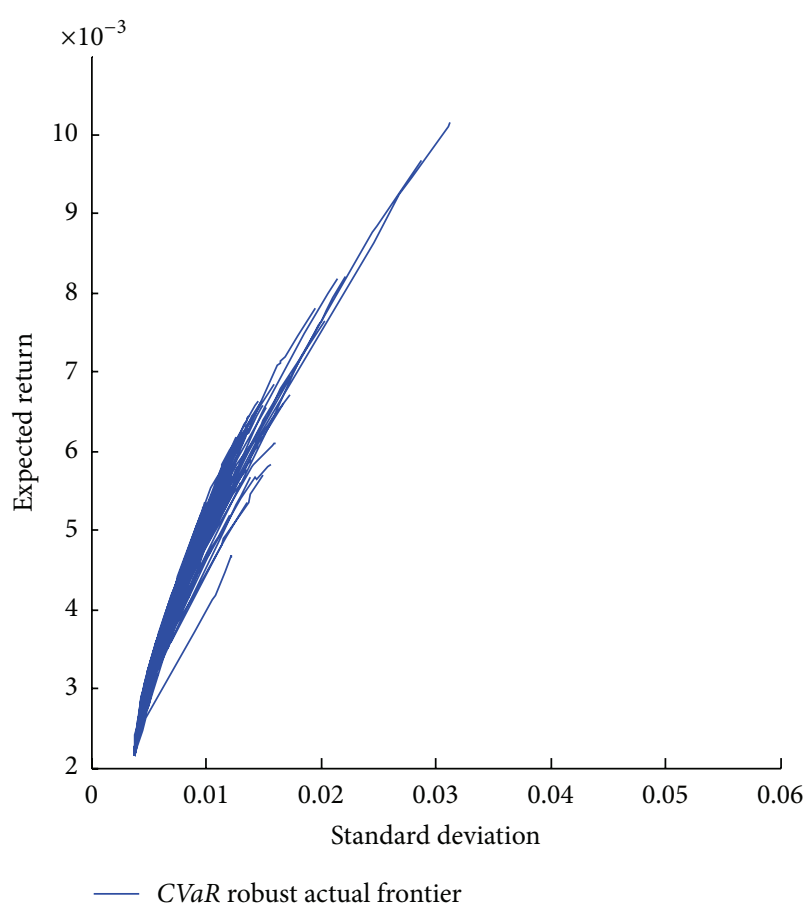

FIgURE 2: $100 C V a R$ robust mean-CVaR actual frontiers ( $\beta=90 \%)$.

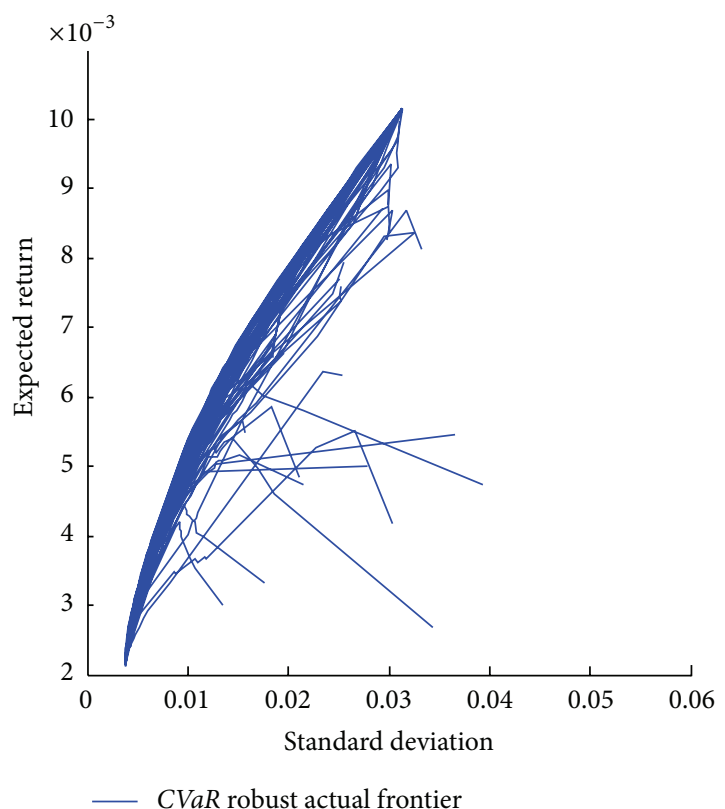

FIGURE 3: $100 C V a R$ robust mean-CVaR actual frontiers $(\beta=75 \%)$.

Considering these figures, when the expected return value increases from left to right, the allocated assets in the portfolios with minimum expected return are replaced by a composition of other assets, gently. Observing the right-most end of each graph, we can conclude the composition of the assets of the portfolio achieved from $C V a R$ robust mean$C V a R$ model with $\beta=99 \%$ is more diversified than that achieved from other models.

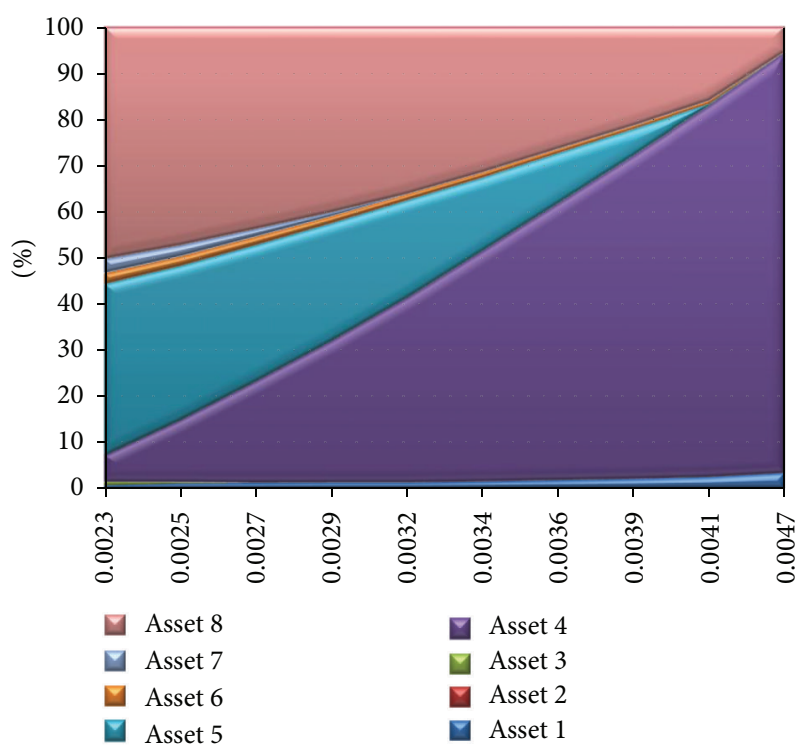

FIgURE 4: Composition of robust mean-CVaR portfolio weights with interval uncertainty set.

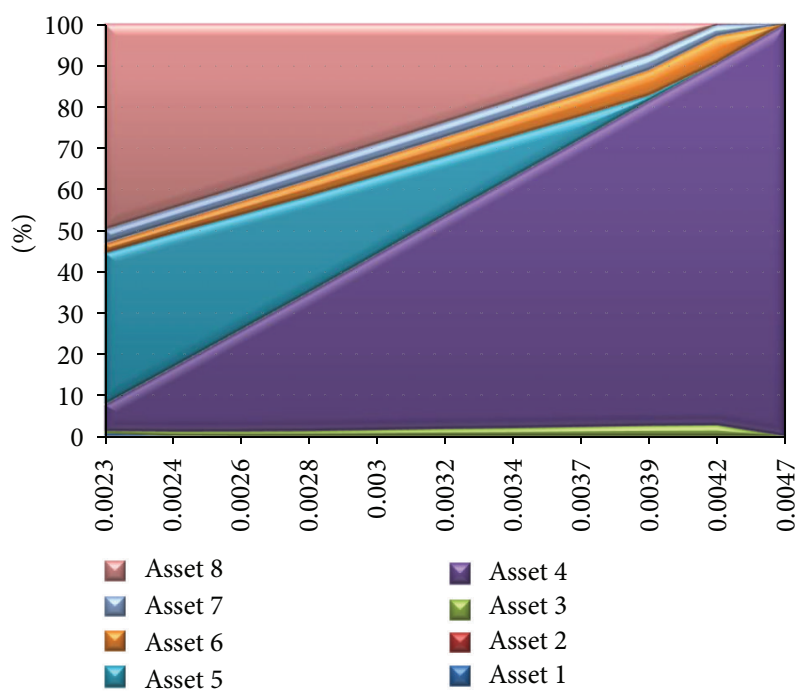

Figure 5: Compositions of robust mean-CVaR portfolio weights with ellipsoidal uncertainty set.

In Figures 9, 10, and 11, the $C V a R$ robust mean-CVaR actual frontiers for different values of $\beta$ are compared with robust mean-CVaR actual frontiers with interval and ellipsoidal uncertainty sets and mean-CVaR true efficient frontier. Since portfolios on the robust mean-CVaR actual frontiers with interval and ellipsoidal uncertainty sets are less diversified, they should accept more risk for a given level of expected return and also achieve a lower maximum expected return. Consequently, in the figures, their actual frontiers are more right and lower than the other frontiers and this is one of the disadvantages of the low diversification in the portfolio. Seeing these frontiers, we also deduce that the maximum expected return and the associated return 


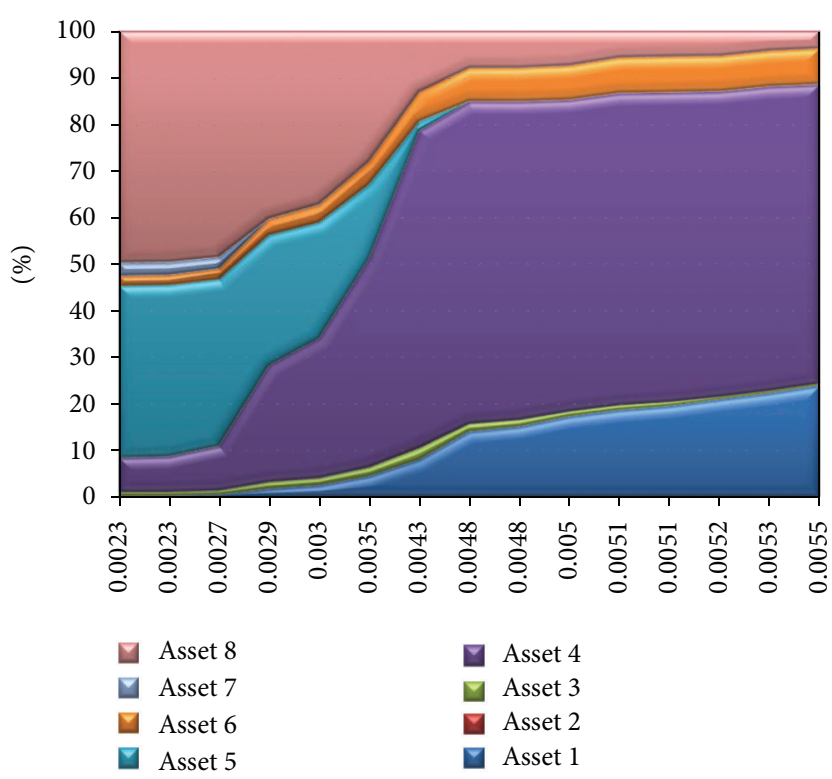

Figure 6: Compositions of $C V a R$ robust mean-CVaR portfolio weights $(\beta=99 \%)$.

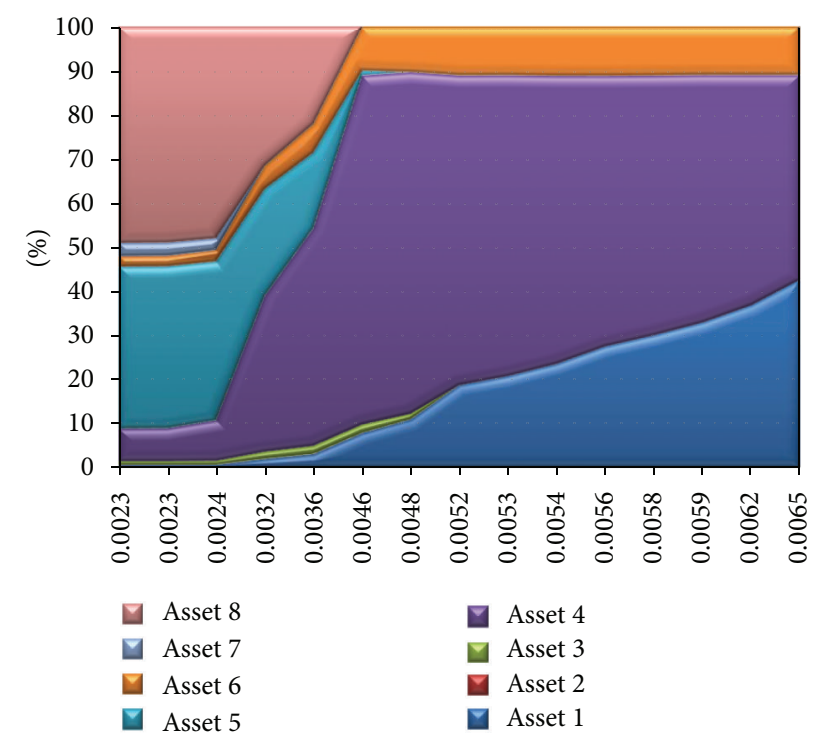

FIGURE 7: Compositions of $C V a R$ robust mean-CVaR portfolio weights $(\beta=90 \%)$.

risk increase as the confidence level $\beta$ decreases. But in this case, the variations on the compositions of the resulting maximum-return portfolios might be large, and so the exact solution will not be always achieved. Instead, the maximum expected return of the portfolio is low for $\beta=99 \%$, and the variations will be low. So, the probability of having poor performance of the portfolio will be reduced when there is a big estimation risk of $\mu$. Thus, resulting robust portfolios will be too conservative. Consequently, an investor who is more risk averse to estimation risk selects a larger $\beta$ and obtains a more diversified portfolio. This justifies that it is reasonable to regard $\beta$ as a estimation risk aversion parameter.

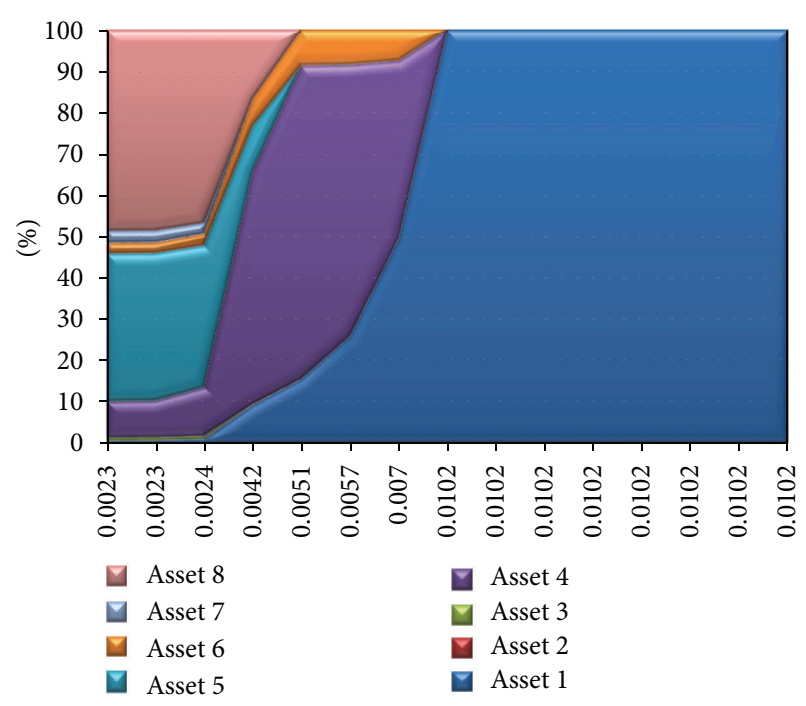

Figure 8: Compositions of $\mathrm{CVaR}$ robust mean- $\mathrm{CVaR}$ portfolio weights $(\beta=75 \%)$.

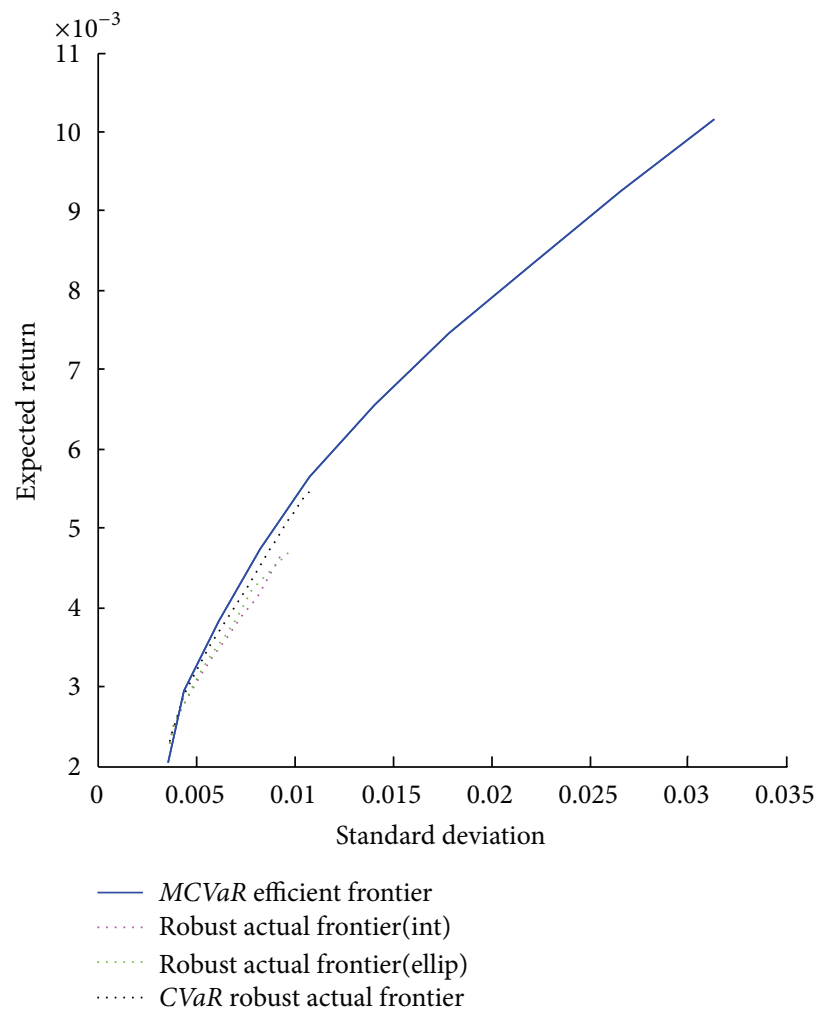

FIGURE 9: Robust mean-CVaR with interval and ellipsoidal uncertainty sets and $C V a R$ robust $(\beta=99 \%)$ actual frontiers.

4.3. Comparison of the Efficiency of Two Approaches for Computing CVaR Robust Portfolios. In Section 3, we introduced two formulations (22) and (26) to compute CVaR robust portfolios. Now, we show that computing $C V a R$ robust portfolios via the smoothing approach (problem (26)) is more efficient. To do so, we compare the time required to solve 


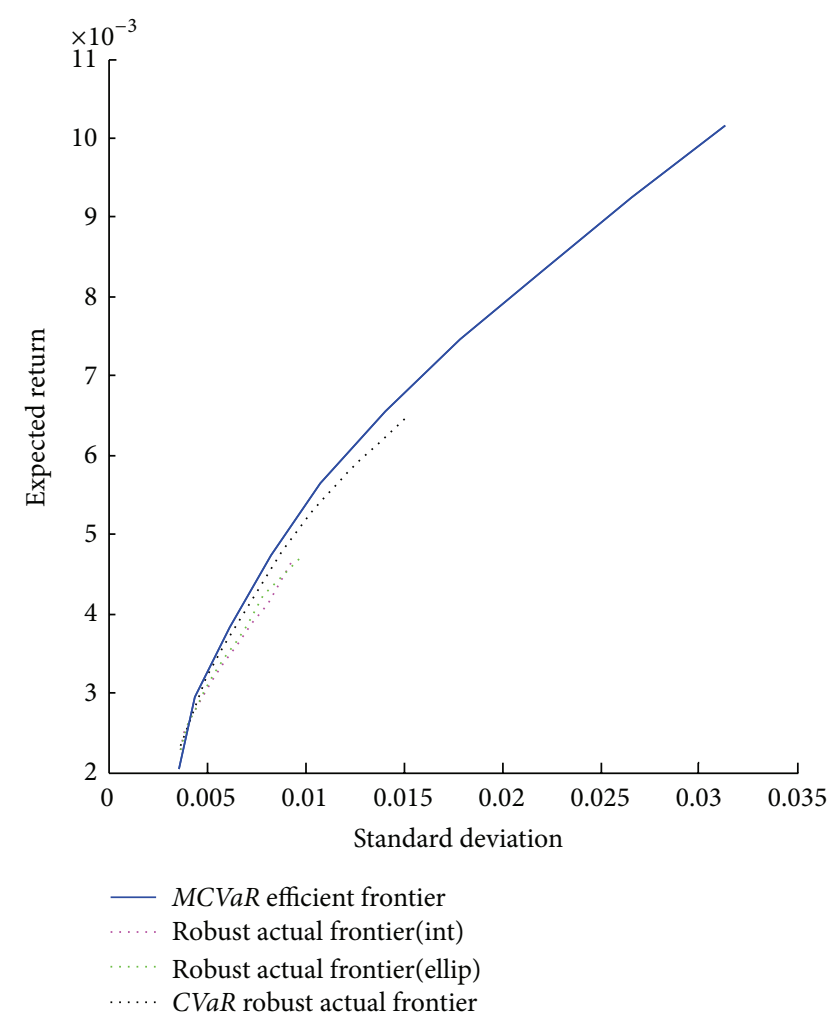

FIGURE 10: Robust mean-CVaR with interval and ellipsoidal uncertainty sets and $C V a R$ robust $(\beta=90 \%)$ actual frontiers.

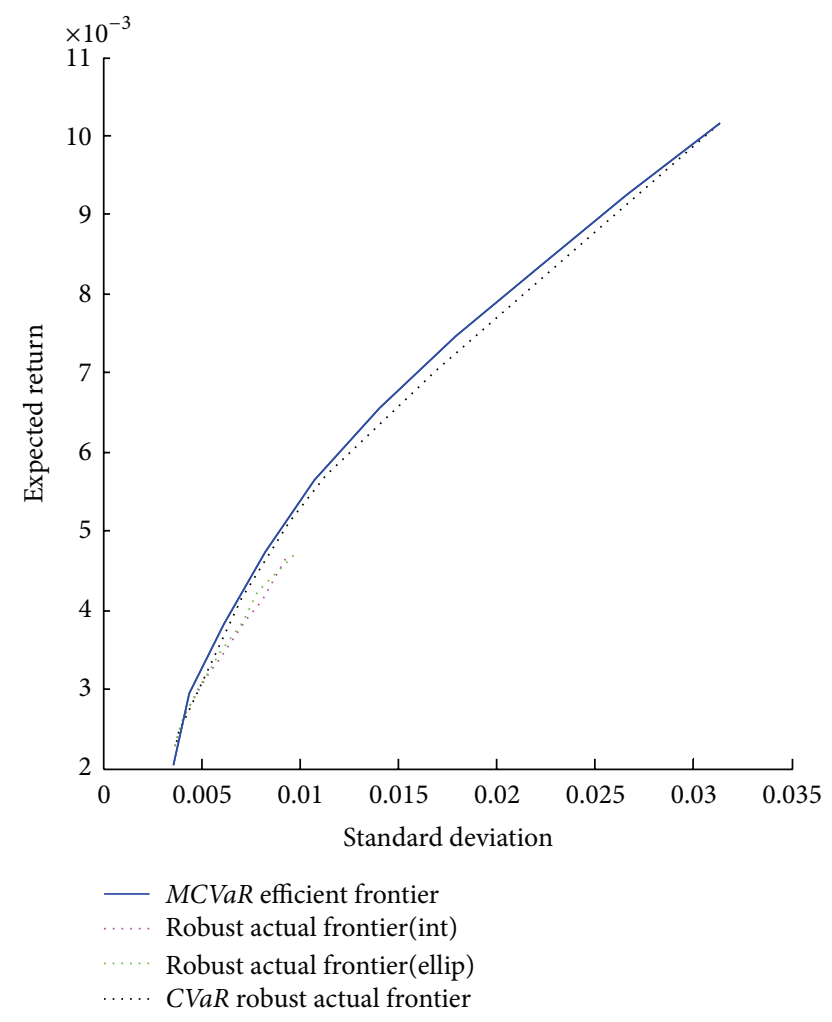

FIGURE 11: Robust mean-CVaR with interval and ellipsoidal uncertainty sets and $C V a R$ robust $(\beta=75 \%)$ actual frontiers. problems (22) and (26) with different number of assets and different number of $\mu$-samples and $y$-scenarios. The results have been given in Table 3.

As we see, the time required to compute $C V a R$ robust portfolios via two approaches differs significantly when the sample size and the number of assets increase. For example, the time required to solve the $C V a R$ robust mean- $C V a R$ problem by two approaches for a problem with 8 assets and 5000 samples and 500 scenarios differs slightly. But, when the number of assets is more than 50 , the number of scenarios is more than 500 , and the sample size is more than 5000, differences become significant. A problem with 148 assets, $3000 y$-scenarios, and $25000 \mu$-samples is solved in less than 80 seconds using smoothing technique, while by (22) it took over 185 seconds. These comparisons show that when the number of scenarios and samples becomes larger, the smoothing approach is more computationally efficient to determine $\mathrm{CVaR}$ robust portfolios than other approach.

\section{Conclusions}

Since risk and return are uncertain parameters in the portfolio optimization, thus their estimation might lead to large error in the final decision. One of the widely used and effective approaches in optimization to deal with data uncertainty is robust optimization. In this paper, we have presented the robust counterpart of mean-CVaR portfolio selection problem under the estimation risk in mean return. We have additionally used $C V a R$ as risk measure to measure the estimation risk in mean return. To solve the model efficiently, the smoothing technique of Alexander et al. [10] is utilized. The performance of the $C V a R$ robust mean- $C V a R$ model with robust mean-CVaR models for both interval and ellipsoidal uncertainty sets is compared. Our experiments have verified that the $C V a R$ robust mean-CVaR portfolios are more diversified. Applying the model for large and real data sets can be considered for future research.

\section{Conflict of Interests}

The authors declared that they have no conflict of interests.

\section{Acknowledgment}

The authors would like to thank office of vice president for research, University of Guilan for supporting this research.

\section{References}

[1] R. H. Tütüncü and M. Koenig, "Robust asset allocation," Annals of Operations Research, vol. 132, pp. 157-187, 2004.

[2] J. Wang, Mean-Variance-VaR Based Portfolio Optimization, Valdosta State University, 2000.

[3] W. N. Cho, "Robust portfolio optimization using conditional value at risk," Final Report, Department of Computing, Imperial College London, 2008.

[4] G. Cornuejols and R. Tütüncü, Optimization Methods in Finance, Cambridge University Press, Cambridge, UK, 2006. 
[5] R. T. Rockafellar and S. Uryasev, "Optimization of conditional value-at-risk," Journal of Risk, vol. 2, pp. 21-41, 2000.

[6] P. Artzner, F. Delbaen, J. Eber, and D. Heath, "Thinking coherently," Journal of Risk, vol. 10, pp. 68-71, 1997.

[7] M. Letmark, Robustness of conditional value at risk when measuring market risk across different asset classes [M.S. thesis], Royal Institute of Technology, 2010.

[8] A. G. Quaranta and A. Zaffaroni, "Robust optimization of conditional value at risk and portfolio selection," Journal of Banking and Finance, vol. 32, pp. 2046-2056, 2008.

[9] P. Krokhmal, J. Palmquist, and S. Uryasev, "Portfolio optimization with conditional value-at-risk objective and constraints," Journal of Risk, vol. 4, pp. 43-48, 2002.

[10] S. Alexander, T. F. Coleman, and Y. Li, "Minimizing VaR and CVaR for a portfolio of derivatives," Journal of Banking and Finance, vol. 30, no. 2, pp. 583-605, 2006.

[11] L. Zhu, T. F. Coleman, and Y. Li, "Min-max robust and CVaR robust mean-variance portfolios," Journal of Risk, vol. 11, pp. 131, 2009.

[12] L. Zhu, Optimal portfolio selection under the estimation risk in mean return [M.S. thesis], University of Waterloo, 2008.

[13] R. O. Michaud, Efficient Asset Management, Harvard Business School Press, Boston, Mass, USA, 1998.

[14] S. Boyd and M. Grant, "CVX users, guide for CVX version 1.21," 2010, http://www.Stanford.edu/ boyd/cvx/.

[15] M. Broadie, "Computing efficient frontiers using estimated parameters," Annals of Operations Research, vol. 45, no. 1, pp. 21-58, 1993. 


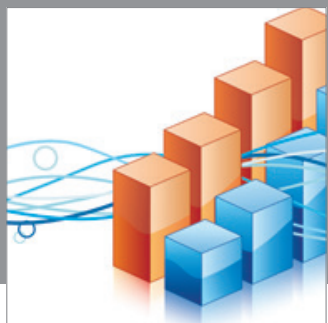

Advances in

Operations Research

mansans

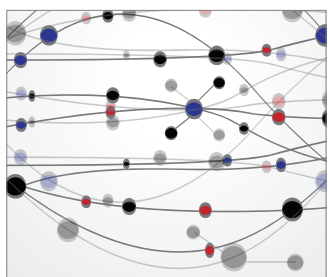

The Scientific World Journal
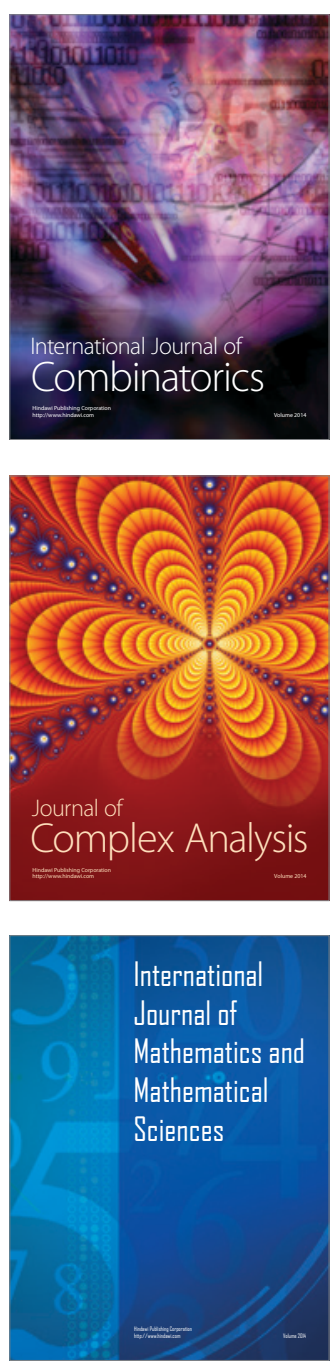
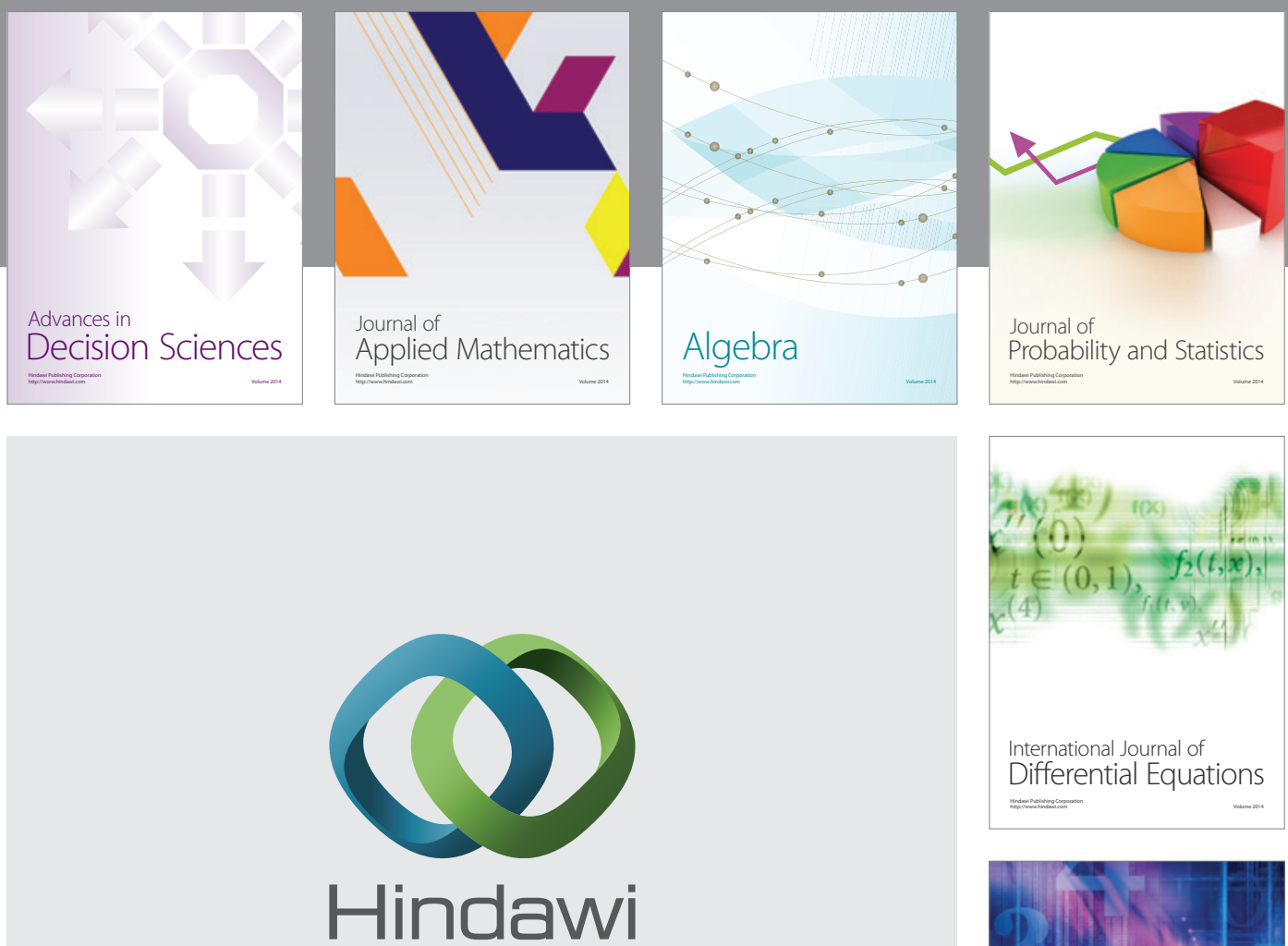

Submit your manuscripts at http://www.hindawi.com
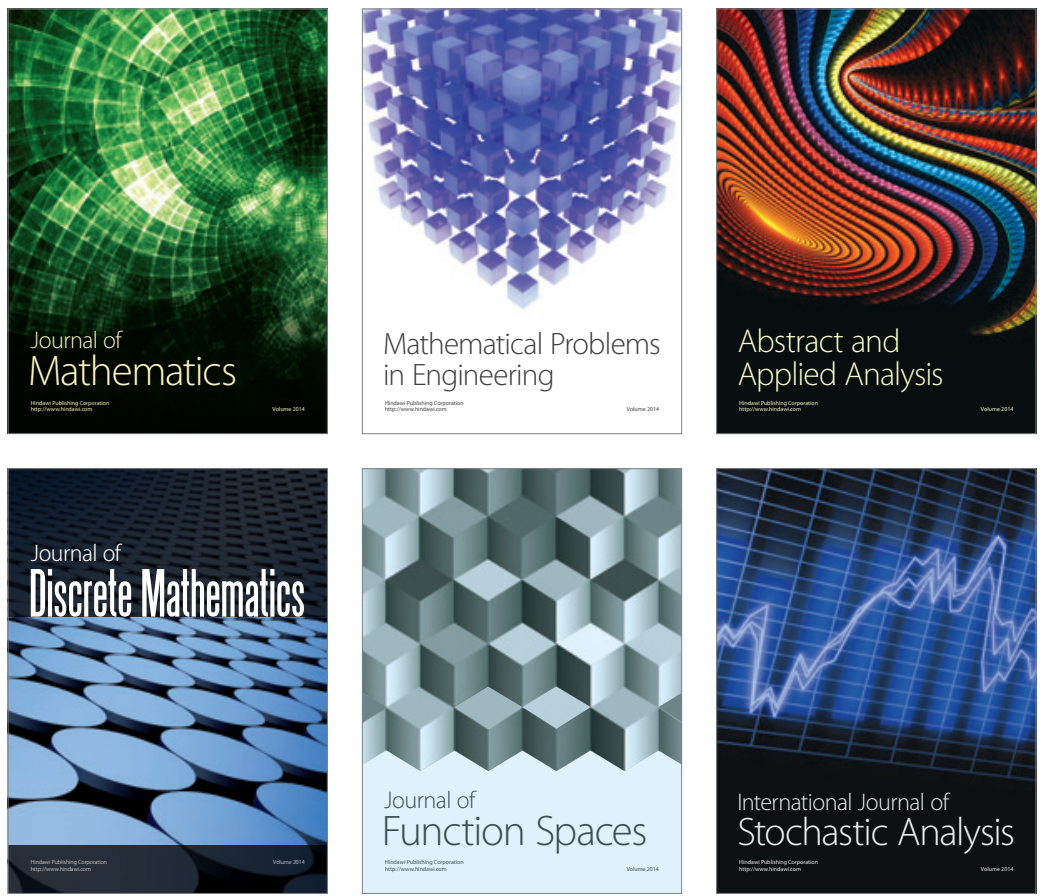

Journal of

Function Spaces

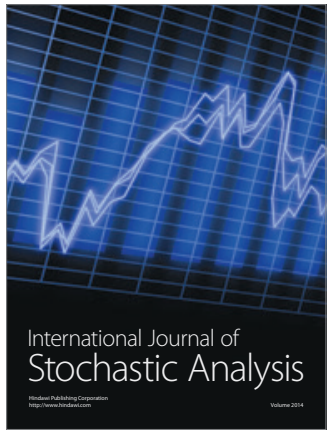

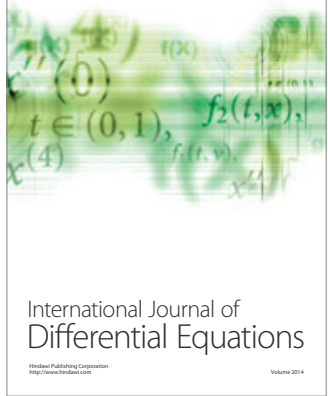
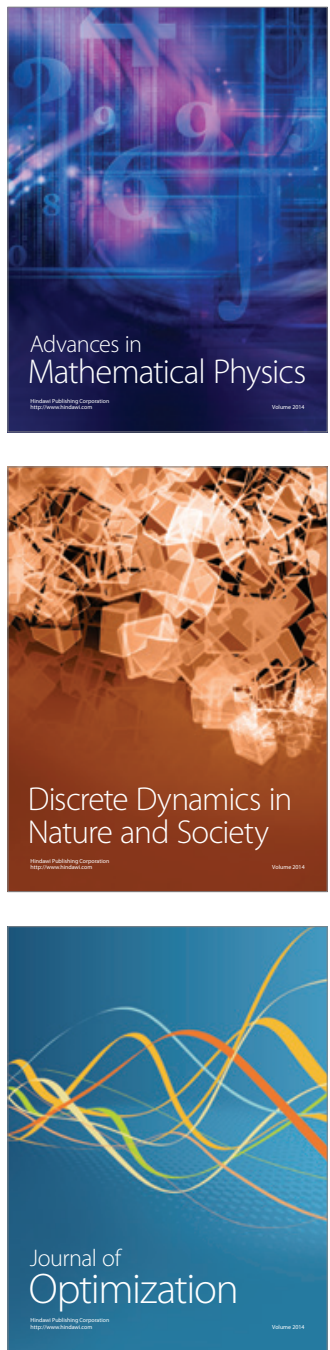